\title{
Formas de evaluación educativa en la Universidad Santo Tomás sede Bogotá, modalidad presencial. Elementos para una caracterización*
}

\author{
Grupo de Investigación Docimófilos
}

Recibido: 2 de marzo de 2009 Revisado: 14 de abril de 2009 Aprobado: 16 de julio de 2009

\section{RESUMEN}

La indagación realizada por el Grupo de Investigación Docimófilos, apuntó hacia una caracterización de las Formas de Evaluación Educativa presentes en la Universidad Santo Tomás Sede Bogotá, Modalidad Presencial. En tal perspectiva, se usó una de las herramientas teórico-metodológicas del arsenal foucaultiano: la arqueología. El estudio se fundamentó en el análisis de diversos discursos -y prácticas- que se han desarrollado en torno del objeto evaluación que se han instalado en la institución, sobre la base de una amplia masa documental. La analítica de la verdad hizo posible la identificación de una pluralidad de modalidades evaluativas que permiten: la producción de saber, la objetivación de las relaciones de poder y la configuración de subjetividades.

Palabras clave: docimología, evaluación, examen, calidad de la educación, prácticas evaluativas, arqueología, verdad, sujeto, subjetividad, historia.

* Este artículo recoge la síntesis de la investigación sobre el objeto evaluación educativa en la Universidad Santo Tomás (Sede Central, Modalidad Presencial). La indagación se llevó a cabo entre febrero de 2007 y junio de 2008; y, se adscribió a la Línea de Investigación Institucional "Cátedra Enrique Lacordaire: Sobre las Libertades y la Educación". Correo electrónico: docimofilos@ yahoo.es. 


\title{
Forms of educational evaluation at the Universidad Santo Tomás, Bogotá branch, on campus modality, elements for a characterization
}

\author{
Docimófilos Research Group
}

\begin{abstract}
The investigation carried out by the Docimófilos Reasearch group, was directed towards a characterization of the Forms of Educational Evaluation that are current at the Universidad Santo Tomás, Bogotá branch, On Campus Modality. In this perspective one of the theoretical- methodological tools from the Foucaultian arsenal was used: Archeology. The study was based on the analysis of diverse arguments and practices that have been developed revolving around the evaluation object that has been installed in the institution., based on a broad documental mass. The analyses of Truth made it possible to identify a plurality of evaluative modalities which permit: the production of knowing, the objectivation of power relationships and the configuration of subjectivities.

Key Words

Docimology, Quality of Education, Evaluative Practices, Archeology, Subjectivity, History.
\end{abstract}




\section{Horizonte de LA InVestigación. A MOdo de INTRODUCCIÓN}

Hay que considerar la obra en su totalidad, seguirla más que juzgarla, recorrer sus bifurcaciones, sus estancamientos, sus ascensos, sus brechas, aceptarla, recibirla entera. De otro modo no se comprende nada.

Deleuze

Puesta en marcha la indagación: Caracterización de las formas "Evaluación Educativa" en la Universidad Santo Tomás Sede Bogotá, Modalidad Presencial ${ }^{1}$ (USTA, 2007, pp. 177-193), se trazó un derrotero que apuntó, en primera instancia, a la apropiación -o mejor, a una aproximación- del instrumental arqueológico y su léxico específico. Fue perentorio, entonces, abordar el análisis de la arqueología del saber, matizada con diversas lecturas y análisis, que han procurado hacerla asequible: Morey, 1983; Martiarena, 1995; Zuluaga, 1987, Guyot, Marincevic y Becerra, 1996, entre otras.

En un segundo momento, dotados ya de algunas herramientas arqueológicas, se realizó una analítica de los documentos institucionales, los cuales señalan múltiples modalidades valorativas, que permanentemente son puestas en escena por diversas instancias y sujetos. Mediante disímiles lecturas, el transcurso por estos momentos y monumentos (documentos) permitió juntar piezas teóricas, anudar sobre los conceptos las mallas del saber (sobre la evaluación y

1 En lo sustentado en el presente artículo, se entiende por: "Caracterización de las formas de Evaluación Educativa, a una descripción histórica, puntual y objetiva - lo efectivamente dicho 0 escrito- referida a las diversas modalidades de evaluación, sus discursos y sus prácticas, contenidas en los archivos documentales - teórico-prácticos- que comportaron los insumos básicos para el desarrollo de la investigación. su praxis en la institución) y desde allí vislumbrar -para futuras indagaciones- las tramas de poder y las constituciones de subjetividad que operan.

El uso de la herramienta arqueológica posibilitó la identificación de enunciados, de modalidades enunciativas o de funciones enunciativas, la ubicación de variadas piezas documentales que contienen regularidades y dispersiones de enunciados, la conformación de los archivos teórico-prácticos -herramientas específicas de la descripción arqueológica-. Todo ello, sumado al saber disperso, a las prácticas conexas o inconexas, a los desarrollos teóricos o metateóricos, a los objetos de análisis (modalidades de evaluación), constituye, como señala Foucault, el a priori histórico sobre el cual es posible concebir la emergencia de un modo específico de saber - para el caso de la investigación, el saber sobre la evaluación educativa y sus formas- en la Universidad Santo Tomás, Sede Bogotá, Modalidad Presencial, desde su refundación hasta el presente ${ }^{2}$.

Dotados del instrumental teórico-metodológico reseñado, fue preciso plasmar el análisis descriptivo -ausente de la interpretación que pretende hacer decir lo que no se dice- en diversos registros, que recapitulan y caracterizan las modalidades de evaluación inherentes a la condición y a la acción de la comunidad académica; de tales registros, este documento es solamente una sucinta muestra. En síntesis, estas etapas constituyeron el horizonte hacia el que apuntó la investigación en su primera etapa.

2 En relación con el estado de la cuestión - objeto de la investigación-, un esbozo inicial se presentó en un artículo publicado tras la aprobación de la propuesta de investigación (USTA, 2007, pp. 186-189); por tal motivo, no hace parte de este documento. 


\section{Condiciones de POSIBILIDAD DE LA PESQUISA}

La indagación fundó su análisis sobre diversas piezas documentales y sus registros, desde la perspectiva de concretar la arqueología como herramienta metodológica que faculta una "historia de las condiciones de posibilidad del saber" (Castro, 2004, p. 32); con una doble intencionalidad: detectar las formas, modos, reglas y juegos como se traman los enunciados (evaluativos) para conformar regularidades, dispersiones y relaciones; y, determinar el conjunto de los elementos que constituyen los archivos -a priori históricos de las condiciones de saberde la evaluación educativa -modalidades, formas y prácticas-, presentes y vigentes en la ubicación y periodización señaladas.

El análisis arqueológico operó sobre unos dominios definidos por las prácticas y los discursos relacionados con un saber en emergencia o en proceso de consolidación; por ello, la materia prima la constituyen los archivos, entendidos como los sistemas generales de formación y transformación de los enunciados (Foucault, 1970); esto es, el conjunto de documentos-monumentos, a través del cual se halla distribuida y dispersa la función enunciativa referida a la evaluación.

La caracterización de las formas evaluativas debió acudir, entonces, a una masa documental constituida por diversos archivos, normativas nacionales, documentos prescriptivos institucionales, producción teórica y metateórica sobre la evaluación, herramientas epistemológica-metodológicas derivadas de los desarrollos foucaultianos y, pruebas testimoniales históricas de sujetos que han jugado diversos roles: docentes, di- rectivos, investigadores, estudiantes, en la Universidad Santo Tomás ${ }^{3}$.

\section{El MÉtodo de LA INDAGACIÓN}

Sé lo que puede tener de un poco áspero el tratar los discursos no a partir de la dulce, muda e intima conciencia que en ellos se expresa, sino de un oscuro conjunto de reglas anónimas

(Foucault, 1970)

La herramienta arqueológica centró su atención en el análisis de los saberes institucionalizados (Zuluaga, 1987), en la descripción de las reglas que para los sujetos, las instituciones, los discursos de un saber específico, "delimitan la práctica del mismo saber en una formación social dada" (p. 28). Esta condición permitió:

- Trazar la historia de los discursos que se han institucionalizado en torno al objeto de evaluación y abordar el análisis de los procesos de epistemologización del saber.

- Analizar las relaciones que se tejen entre discursos (administración, legislación, educación, pedagogía, didáctica, etc.) en orden a la conceptualización de la evaluación.

- Realizar una descripción histórica con el objeto de percibir los tránsitos de la evaluación a través de determinados

3 Al término del análisis de los archivos documentales, el grupo de investigación se percató de un vacío teórico, en razón a la ausencia de documentos institucionales que recogieran la memoria y la historia institucional del periodo comprendido entre 1965 y los primeros años de la década del ochenta. Se debió abordar, entonces, una diligente búsqueda de documentos en diversas instancias: Vicerrectoría Académica, Secretaría General, Biblioteca, Hemeroteca, facultades, departamentos y otras unidades académicas (Oficina de Admisiones, Unidad de Evaluación y Perfeccionamiento Docente, Unidad de Autorregulación y Acreditación); instancias celadoras de la memoria institucional, a la par, de una reconstrucción histórica a partir de la memoria viviente de la Universidad 
umbrales que le han otorgado el estatuto disciplinar.

- Finalmente, señalar los diversos trayectos que tal práctica discursiva ha trazado, por casi medio siglo, en la USTA.

La descripción histórica realizada sobre las formas evaluación educativa demandó, en un primer momento, el establecimiento de las regularidades discursivas -de prácticas evaluativas y de discursos prescriptivos-; desde esa perspectiva fue necesario determinar la unidad (o las unidades) de análisis que yacen presentes en un conjunto de acontecimientos dispersos y que han posibilitado la instalación de la maquinaria evaluativa. Fijadas tales unidades, fue preciso, de inmediato, dejarlas en suspenso, a fin de "restituir al enunciado su singularidad de acontecimiento", mostrar su discontinuidad y "verlo surgir en su irrupción histórica", asistir a su emergencia.

Por otra parte, se analizaron los modos como el discurso evaluativo se institucionalizó, a través del establecimiento de una regularidad entre un grupo de enunciados (dispersos, constantes, coexistentes, persistentes) manifiestos en un conjunto de prácticas y saberes. Asimismo, se examinaron las condiciones de aparición histórica del saber evaluativo, las superficies que lo hacen visible, las instancias que lo delimitan y los modos como se especifica a través de diversas prácticas. Luego, se caracterizaron diversos modos de enunciar la evaluación -el régimen de su enunciación, en su dispersión-, a través de la determinación del estatuto y la posición de los sujetos legisladores, teóricos, técnicos, evaluados, evaluadores, metaevaluadores, directivos, administrativos, etc. - y de los ámbitos institucionales e interinstitucionales a través de los cuales se formula. Posteriormente, se escudrinaron las superficies en que aparecen y circulan conceptos y formas evaluativas, sus modos de sucesión -encadenamiento de enunciados-, sus formas de coexistencia y los procedimientos a través de los cuales se concretan. Finalmente, se determinó, el modo como la evaluación se configura en estrategia -organizaciones de conceptos, agrupamientos de objetos, tipos de enunciación, que forman, de modo coherente riguroso y estable, temas, grupos de temas, positividades o teorías.

Una segunda instancia de la descripción consistió en la conformación y caracterización del enunciado y del archivo. Para ello se precisó fijar la terminología arqueológica -enunciado, discurso, formación discursiva- (Foucault, 1970, pp. 129-223). Tras el análisis documental, se constató la dispersión del enunciado evaluación, bajo diferentes apariencias y nominaciones, refiriéndose, incluso, a operaciones distintas y contradictorias. Tal análisis desestimó tres cuestiones: ¿quién ha dicho tal enunciado?, ¿qué quería decir?, ¿qué posición ocupa el sujeto (evaluador-evaluado) frente al enunciado?; la pesquisa atendió efectivamente a la inscripción de lo que se dice, sin buscar en lo dicho un no-dicho (hermenéutica) o un sobre/entre dicho (formalización). En síntesis, la descripción del enunciado evaluación constituyó una analítica histórica desarrollada, como señala Foucault, fuera de toda interpretación.

Este segundo momento, permitió mostrar el modo como se puede organizar un discurso (evaluativo): dominio conformado por los enunciados, sus formas de agrupamiento, las unidades que constituyen y los métodos que permiten describirlo (p.193). Tal domi- 
nio implica dos nociones mayúsculas que constituyen el objeto del análisis arqueológico: la función enunciativa y la formación discursiva, cuyos elementos nodales son el enunciado y el archivo. Para el análisis arqueológico, los enunciados son acontecimientos y cosas (p. 218); al conjunto de acontecimientos y cosas, articulado según sus a prioris históricos, se denomina archivo: "sistema general de enunciabilidad y de funcionamiento de los enunciados" (Morey, p. 214); "el sistema general de la formación y la transformación de los enunciados" (Foucault, 1970, p. 221). El archivo constituye el dominio específico de la arqueología, que designa:

[...] el tema general de una descripción que interroga lo ya dicho al nivel de su existencia: de la función enunciativa que se ejerce en él, de la formación discursiva a que pertenece, del sistema general de archivo del que depende. La arqueología describe los discursos como prácticas especificadas en el elemento del archivo (p. 223).

Lo referido señala cómo y por qué la arqueología permite abordar desde un campo epistémico el surgimiento y la consolidación de los discursos, la convergencia en las prácticas sociales y de los usos conferidos a la evaluación, en la Universidad Santo Tomás. De este modo, coincidiendo con Zuluaga (1987, p. 16), la caja de herramientas brinda la "posibilidad de historias particulares, de historias diferenciales, para saberes que, a pesar de su dispersión [heterogeneidad, incluso ambigüedad] obedecen a regularidades conformadas por las prácticas discursivas y las prácticas sociales".
En consecuencia, cualquier historia que describa la arqueología, tendrá como fundamento la interrogación por las formas, como, la verdad ha ocupado un lugar relevante en las sociedades. Una historia de este talante no se ocupa de validar o de invalidar la verdad del saber, sino de indagar "por los procedimientos de su fundación, las reglas a las que obedece y las leyes de su funcionamiento". Una historia de las estrategias de la verdad busca describir las formas como la verdad emerge, no se detiene en lo que pueda haber de cierto en los conocimientos, sino en el análisis de los "juegos de verdad", en que los sujetos y los saberes se reconfiguran constante y perpetuamente.

\section{Analítica de las formas EVALUACIÓN EDUCATIVA}

La analítica histórica abordó una descripción arqueológica de los enunciados que se relacionan con las diversas formas de evaluación educativa, en su regularidad y en su dispersión, prescritas y manifiestas en y a través de los documentos institucionales: Estatuto Orgánico, Proyecto Educativo Institucional; Política Curricular, Estatuto Docente, Reglamento General, Política de Evaluación Docente; Política Curricular, Política de Autorregulación y Acreditación, Política de Admisiones y otros documentos institucionales. Sin forzar significaciones y búsquedas de sentido, apartándonos -en lo más posible- de cualquier toma de postura, rehusando el recurso hermenéutico e interpretativo, con el propósito de tratar, como sugiere la arqueología, el documento como monumento. 


\section{Formas evaluación educativa que prescribe el Estatuto Orgánico}

El Estatuto Orgánico de la Universidad Santo Tomás ${ }^{4}$ prescribe un conjunto de pautas relativas a diferentes formas evaluación educativa (estilos, prácticas y modalidades), a través de las cuales se constata el cumplimiento -con criterios de calidad- de la misión, de los propósitos y objetivos institucionales. El documento señala que, dado que el Estado, a través de la jurisprudencia, reconoce la autonomía de las instituciones, la USTA, goza (2002, p. 18), de

[...] plena facultad para darse y modificar sus estatutos y reglamentos, designar sus autoridades académicas y administrativas, crear, organizar y desarrollar sus unidades y programas académicos, definir y organizar sus labores formativas, científicas, culturales y de extensión, otorgar títulos, establecer sus propios criterios y mecanismos de selección de los profesores y estudiantes y aplicar sus recursos para el cumplimiento de su misión institucional y de su función social.

4 El Estatuto Orgánico es el documento institucional que determina y expresa la naturaleza y razón de ser de la Universidad (misión, principios, objetivos y funciones sustantivas; órganos de gobierno, dirección y administración; composición y distribución territorial; organización académica, investigativa, extensión y bienestar universitario; agentes, acciones, procesos y proyectos; intencionalidades, etc.). La primera versión del Estatuto Orgánico fue aprobado por el Consejo Superior de la Universidad Santo Tomás "mediante el acuerdo No. 6 Bis del 14 de junio de 1972". El título IV, Del Gobierno de la Universidad, explicita las autoridades instancias colectivas o individuales que regentan los destinos de la institución, dentro de cuyas funciones se pueden contar diversas modalidades evaluativas que, de modo directo 0 indirecto, relacionan los procesos de enseñanza-aprendizaje 0 determinan los fines a los que se orienta la Universidad. Muchas de estas prácticas evaluativas, son recogidas en los documentos vigentes actualmente (USTA, 1972, pp. 3-24). La versión actual del Estatuto Orgánico de la Universidad Santo Tomás fue aprobada por el Ministerio de Educación, mediante la Resolución 3149 del 17 de diciembre de 2001 y promulgada a la comunidad académica el 11 de julio de 2002, momento a partir del cual entra en vigencia y deroga las disposiciones anteriores sobre tal materia (USTA, 2002, pp. 3-4, 91).
El cumplimiento de los principios institucionales, de los objetivos y de las funciones sustantivas demanda, por parte de las instancias responsables, la adopción de diversos mecanismos conexos con modalidades o prácticas valorativas-evaluativas: selección, incorporación, vigilancia, regulación, control, agenciamiento, promoción, adopción de metodologías apropiadas, aprovechamiento de recursos, divulgación, identificación, perfeccionamiento, cualificación, actualización, vinculación (de agentes, objetos, programas y procesos). Todas estas acciones constituyen procedimientos evaluativos a través de los cuales se concreta y se determina la calidad del ejercicio de las funciones sustantivas que orientan la vida universitaria.

En términos generales, es posible identificar en el Estatuto Orgánico, específicamente en lo relativo a las funciones de gobierno y administración de la institución, un conjunto de prácticas y discursos que remiten a procedimientos evaluativos, los cuales, directa o indirectamente, inciden en los procesos de enseñanza-aprendizaje, como determinar políticas institucionales; aprobar reformas estatutarias, reglamentos y disposiciones; creación o supresión de unidades (docentes, investigativas, administrativas, de bienestar); dirigir, estudiar y resolver asuntos académicos; adopción y reforma de programas; determinar calendarios académicos, sistemas de evaluación, calificación y métodos de docencia e investigación; elaborar y aprobar estatutos y reglamentos generales y específicos (de docentes, de estudiantes, de facultades, de los institutos y de los departamentos); elaborar y aprobar planes de financiación y presupuestos; elaboración, aprobación y modificación de los pénsum y programas académicos; proponer proce- 
dimientos para la capacitación y promoción del profesorado; determinar estímulos y distinciones para la motivación de los estudiantes; evaluación periódica de planes y programas de estudio y métodos de enseñanza utilizados; etc.

Todos estos procedimientos implican, a la vez: sujetos (evaluados y evaluadores); objetos de saber y campos de conocimiento (disciplinas, áreas, asignaturas) sometidas también a procedimientos evaluativos; instituciones y relaciones internas y externas en las que se ponen en juego mecanismos de confrontación y evaluación; acciones, procesos, proyectos, programas, etc., que han de ser sometidos a instancias de control o regulación acciones; en fin, múltiples y diversos modos de percibir la realidad y de ser en el mundo, y de someterlos a juicio, valoración y medida.

\section{Modalidades evaluativas que explicita el PEI}

La carta de navegación institucional ${ }^{5}$ señala la relevancia de los procedimientos eva-

5 El marco legal de los PEl se fundamenta en la Ley 115 de 1994 (Artículo 73), en la cual se prescribe que "con el fin de lograr la formación integral del educando, cada establecimiento educativo deberá elaborar y poner en práctica un Proyecto Educativo Institucional en el que se especifiquen entre otros aspectos, los principios y fines del establecimiento, los recursos docentes y didácticos disponibles y necesarios, la estrategia pedagógica, el reglamento para docentes y estudiantes y el sistema de gestión". El PEl primera versión fue propuesta Cárdenas y Rodríguez (1996), como documento-taller que sería discutido por la comunidad académica en sus diferentes instancias. Una segunda versión, fruto de la recolección de información del trabajo anterior, el cual es presentado por un comité conformado para fines de revisión del documento anterior y encargados de recoger las inquietudes presentadas por la comunidad universitaria; este trabajo se da a conocer a la comunidad académica en 1999. La tercera versión (actual), presentada en 2004 como trabajo compilado de los anteriores, presenta algunas observaciones derivadas del avance propio de la universidad y de los trabajos adelantados desde la perspectiva de los procesos de acreditación. luativos que acompañan toda la actividad académica. La evaluación constituye un aspecto fundamental en la gestión académica, compete a directivos, administrativos, docentes y estudiantes. Es utilizada como instrumental para valorar resultados, diagnosticar procesos, pronosticar, interpretar, redireccionar, corregir, promover, seleccionar, etc.

La formulación de la misión comporta una modalidad explícita de evaluación, al referirse, en su intencionalidad, a la promoción de la formación integral de las personas. Este término promoción es eminentemente valorativo-evaluativo: "Promover es elevar hasta la armoniosa integración de ciencia y conciencia" (USTA, 2004c, p. 24). Asimismo, el modo de responder de la Universidad a las exigencias y desafíos de la sociedad supone una función cualificada y cualificadora, por cuanto la ética, la creatividad, la crítica y la reflexión han de permear el accionar de los egresados:

Las personas de los estudiantes se harán aptas y competentes para responder a las exigencias de la vida humana, tanto personal como colectivamente. Esa capacidad de respuesta deberá ser "ética", es decir, con disposición para el control reflexivo y acción valiosa frente a las distintas propuestas morales vigentes (p. 28).

Los objetivos estatutarios develan formas de evaluación que constituyen mediaciones y el instrumental garante del mejoramiento de la calidad de la institución. Las prácticas valorativas son el horizonte orientador del telos al que apunta toda la acción institucional (la formación integral); el mecanismo determinante para la selección y adopción de un currículo preciso; el dispositivo de funcionamiento para la apertura, la perti- 
nencia y la flexibilidad curricular; la maquinaria que permea constantemente las funciones sustantivas (docencia, investigación y proyección social) para cualificarlas; la instancia reguladora de las políticas institucionales; en síntesis, es el arsenal teórico que determina las bondades y la relevancia de los aportes institucionales al conjunto de la sociedad a la que sirve. "Es preciso, señala el documento, evaluar continuamente la interacción con el medio externo y discernir su incidencia en la docencia, investigación y proyección social"' (p. 103).

De modo concreto, el PEI prescribe modalidades de evaluación de procedimientos educacionales: en primera instancia, señala los criterios de autoevaluación y autorregulación institucionales; en segundo lugar, los criterios de evaluación de estudiantes y docentes (pp. 139-142).

- Criterios de autoevaluación y autorregulación. Mecanismos imprescindibles para la consecución de la "autocomprensión permanente" que exige la adopción de mecanismos de autoevaluación o valoración (evaluación) de la calidad de su acción interno y de su influjo externo.

Cuando se evalúa la calidad, señala el PEI, es necesario introducir elementos de comparación y contraste, que no están fuera de la institución, sino en la formulación de la propia Misión, en el Proyecto Educativo Institucional acerca de los antecedentes históricos, propósitos generales, formación integral, funciones sustantivas, comunidad educativa, estructura organizacional, interacción con el medio externo, administración y gestión (p. 140).

La autoconfrontación se efectúa sobre la base de unos indicadores (positivos, de avances y realizaciones; o, negativos, de retroceso o parálisis), a través de los cuales, "se logran referentes concretos para tomar decisiones de autorregulación y planeación redireccionante".

- Criterios de evaluación de estudiantes y docentes. La evaluación es una mediación pedagógica que acompaña permanentemente todos los procesos de enseñanza-aprendizaje "no con el propósito de excluir, sino de promover". Actualmente, en la acción educativa, se ha ingresado en una cultura en la cual "todo se evalúa": en principio, se evaluaban los contenidos y los objetivos de los programas; posteriormente se pusieron de moda los logros y se valoraban según la prescripción de unos indicadores (homogéneos) que medían el alcance (o no alcance) de aquéllos; en los últimos lustros se ha instalado el discurso de las competencias que se han constituido en el nuevo objeto de evaluación.

Se valoran los logros de aprendizaje -señala el PEI- con el objeto de que los estudiantes se hagan cargo de su propio ascenso en el desarrollo de competencias, de acuerdo con los estándares que impone el currículo, en función de la formación integral. La evaluación proporciona sentido humano a toda la actividad educativa y condiciona la vida futura de nuestros estudiantes. Mediante la evaluación, cada uno juzga qué nivel de maduración ha alcanzado. Por otra parte, la evaluación indica en qué sentido se deben redireccionar el proceso de enseñanzaaprendizaje y el tipo de interacción entre los sujetos de éste: estudiantes-docentes-directivos-administrativos-mediaciones pedagógicas" (p. 141). 
En lo relativo a la evaluación docente, el PEI, refiriéndose a lo prescrito por el Estatuto Docente, establece (p. 142):

La evaluación del docente se considera un proceso integral y se constituye en un espacio académico de reflexión, construcción y propuesta de mejoramiento continuo, que busca, mediante la autoevaluación, la coevaluación y la heteroevaluación, promover el ejercicio de la docencia calificada a través de estrategias de participación, diálogo y concertación entre los diversos actores del proceso educativo: docentes, estudiantes y directivos (Artículo 27).

Finalmente, es preciso señalar que diversos procesos de regulación, control y gestión están atravesados por instancias valorativas y cualificadoras que constituyen, en cuanto tales, procedimientos de evaluación que, de una u otra manera, inciden el accionar de la institución. La gestión desarrollada por cada una de las unidades académicas, desde la perspectiva de la adopción de la cultura de la calidad demanda periódicos ejercicios de evaluación, regulación y control; esto es, como señala Foucault, el ingreso a procedimientos de examinación perpetua, de seriación y clasificación; de evaluación permanente:

Anualmente, a las personas responsables de diferentes niveles de gestión institucional se les pide información verificable y definir logros, debilidades y factores incidentes. No solamente se evalúa la gestión global, propiamente dicha, sino que también se invita a la autoevaluación del desempeño personal en las funciones administrativas y en la función docente e investigativa. Todo esto se completa con la práctica de la héteroevaluación y coevaluación por los estudiantes y usuarios de los servicios institucionales (p. 156).

\section{La evaluación prescrita en la Política Curricular}

En tanto dispositivo orientador y directriz del accionar de los programas, planes de estudio, currículos; del desarrollo y aplicación de los proceso formativos propios de la Institución y de todos sus miembros, la Política Curricular (USTA, 2004a) constituye una enjambrazón de procedimientos evaluativos, en la medida en que articula el conjunto del accionar de la Universidad. La intencionalidad de esta política contiene parámetros valorativos, "orientaciones y directrices" que rigen el "desarrollo y aplicación de los procesos formativos propios de la Universidad"; del mismo modo, signan “el ejercicio de la actividad académica de todos los miembros de la comunidad universitaria". Es aquel el ente legitimador de las "orientaciones" y de las "directrices", y el "guía" validador del "ejercicio de la actividad académica de todos los miembros de la comunidad universitaria". Y todo esto para cumplir con el referente institucional mayúsculo inspirador de todos los demás: la misión de la Universidad, en la intencionalidad de lograr los "objetivos propios de la institución y la realización de su proyecto educativo". Por todo lo anterior, es la Política Curricular la que articula y dinamiza el "conjunto de criterios" los "planes de estudio, programas, metodologías y procesos" $\mathrm{y}$ todo lo que ayuda a "llevar a cabo el Proyecto Educativo Institucional".

La apuesta institucional (formación integral), aparejada de un plexo de valores en el itinerario hacia la construcción de la verdad (Facientes Veritatem) suponen un tipo de educación atravesada de modo permanente por componentes valorativos y procedimientos evaluativos y examinatorios, que 
desde los más elevados índices de calidad propenden a la constitución del conocimiento, del saber, de la ciencia, de las artes y la filosofía, en fin, de las posibilidades y condiciones del intelecto humano.

El documento, específicamente numeral 6.3 (pp. 48-49), se ocupa de la prescripción de los aspectos de la evaluación curricular, que debe ser integral, permanente, omniabarcante, axiológica, participativa, dialógica, diagnóstica, procesual, comunicativa, multifuncional..., y, a través de diversos procedimientos:

La evaluación curricular debe ser integral, en cuanto involucra el proceso de enseñanza-aprendizaje, el desempeño profesional del docente y los programas académicos. La evaluación debe vincular los principios y lineamientos del enfoque pedagógico de la Universidad propuestos en el PEI, reconociendo además, desde la concepción de evaluación, un proceso participativo y dialógico a partir de la identificación de fortalezas y debilidades que favorezcan consolidar el mejoramiento de la calidad académica.

El proceso de evaluación docente y de estudiantes toma en consideración tres momentos: autoevaluación, coevaluación y heteroevaluación. La autoevaluación se constituye en el espacio de reflexión del docente o del estudiante sobre el proceso de enseñanza-aprendizaje, respectivamente, mediado por el juicio crítico y la ponderación ética; la coevaluación es la posibilidad de ser evaluado entre pares y la heteroevaluación es la mirada externa del proceso por parte de los docentes hacia los estudiantes y viceversa, como también desde los directivos de programa.
Así como los aspectos curriculares suponen diversos procedimientos de evaluación, los aspectos administrativos y financieros comportan múltiples modalidades evaluativas en orden a elaboración de portafolios de servicios, inventario de cursos, programas de cooperación entre unidades académicas, proyecciones sobre uso de espacios físicos y recursos, contrataciones, etc.

\section{Formas evaluación educativa que prescribe el Estatuto Docente}

El Estatuto Docente (USTA, 2004b) es el documento institucional que, en consonancia con el Estatuto Orgánico y las normas vigentes de la Educación Superior, prescribe los "principios y normas básicas que regulan las relaciones entre la Universidad y sus docentes, señalan los deberes y derechos, las modalidades de vinculación, ingreso, escalafón, desempeño, permanencia, promoción, régimen disciplinario y retiro" (p. 9). Los principios (potencialidades, cualidades, capacidades, disposiciones), consagrados en la norma como requisitos del docente, constituyen, en primera instancia, mecanismos de selección determinantes para que un profesional pueda ser (si cumple los requisitos), miembro de la comunidad académica de la USTA (p. 11).

La vinculación de docentes a la institución, en cualquiera de sus modalidades y su ubicación en alguna de las categorías facultadas por el Estatuto (pp. 11-12) implican necesariamente procedimientos evaluativos: diligenciamiento de un formato (hoja de vida) y la constatación mediante documentos adjuntos, de la formación requerida; examinación, certificación y comprobación de la apropiación de saberes, habilidades 
y dominios según el cargo; enjuiciamiento y validación de tales condiciones mediante entrevista con agentes administrativos (Decano de Facultad y División); aval, presentación del candidato por estas instancias y por la Vicerrectoría Académica a la Rectoría, para su inclusión en la nómina laboral y aprobación definitiva ${ }^{6}$.

La promoción de un docente a una categoría del escalafón obedece a la práctica de diversos mecanismos de evaluación que determinan el cumplimiento (o incumplimiento) de los requisitos exigidos por la categoría correspondiente: posesión de títulos; experiencia docente o profesional cualificada y comprobada; experiencia directiva, administrativa o académica en la USTA; seminarios de inducción docente; evaluación docente integral; producción intelectual calificada, aceptada y certificada por pares académicos (p. 17). Los requisitos que han de acreditarse para el ingreso, el ascenso o la homologación en el escalafón, según la categoría, son los criterios (indicadores) de clasificación (evaluación) docente (Art. 1718). En todo caso, "el ascenso de categoría debe estar soportado por el proceso de evaluación integral satisfactorio del desempeño como docente" (p. 19).

La instancia de meta-evaluación suprema de la institución la constituye un Comité de Promoción y Ascenso, su finalidad consiste en "estudiar y resolver las solicitudes de ascenso de los docentes presentadas por el Consejo de Facultad o por los consejos res-

6 La firma del contrato es el aval definitivo, el cúlmen del proceso de selección e inclusión, toda vez que se han satisfecho las demandas del perfil docente. El docente es adscrito a una facultad 0 unidad académica específica sin excluir la vinculación a cualquier otra en la que pueda prestar sus servicios, según las necesidades. pectivos" (p. 21)7. Para entonces, el procedimiento de selección para ascenso ha debido pasar por otros entes examinatorios (Secretaría de Facultad, Consejo de Facultad o Unidad Académica), instancias que analizan la documentación, asignan el puntaje y emiten su concepto valorativo, que "será remitido al Comité de Promoción y Ascenso para su pronunciamiento", previo cumplimiento de los requisitos y sustentación, mediante los documentos correspondientes (p. 22).

Además, el documento prescribe unas prácticas evaluativas concretas, desde la perspectiva de clasificar a los docentes en las diversas categorías del escalafón: Evaluación docente anual; Producción intelectual, escritos científicos, técnicos, literarios y humanísticos; Obras artísticas; inventos, diseños y desarrollos tecnológicos originales; segundos títulos; distinciones académicas; desempeño de cargos directivos en la USTA; investigación institucional; investigación externa comprobada y reconocida. Para efecto de ascenso, prescribe el Estatuto, la puntuación (calificación) de cada ítem se realiza por una sola vez (pp. 23-24).

De modo específico, el Capítulo VIII del Estatuto se refiere a los mecanismos de evaluación docente: "un proceso integral y se constituye en un espacio académico de reflexión, construcción y propuesta de mejoramiento continuo que busca, mediante la autoevaluación, la coevaluación y la heteroevaluación, promover el ejercicio de la

7 El Comité de Promoción y Ascenso está conformado por: el Vicerrector Académico General o su delegado o la máxima autoridad académica de la sede respectiva, quien lo convoca y preside; un representante del Rector; un Decano de División elegido por sus pares; un Decano de Facultad elegido por sus pares; un representante de los docentes elegido por sus pares; un representante del Consejo Administrativo-Financiero Particular. 
docencia calificada a través de estrategias de participación, diálogo y concertación entre los diversos actores del proceso educativo: docentes, estudiantes y directivos" (p. 29); señala además, como función de la Unidad de Planeación Académica y Perfeccionamiento Docente, el establecimiento de procesos y procedimientos necesarios para la aplicación de los mecanismos de evaluación docente ${ }^{8}$.

\section{Formas evaluación educativa que dispone el Reglamento Estudiantil}

Según lo establece la Ley 30 de 1992, “Las instituciones de Educación Superior deberán tener un reglamento estudiantil que regule al menos los siguientes aspectos: requisitos de inscripción, admisión y matrícula, derechos y deberes, distinciones e incentivos, régimen disciplinario y demás aspectos académicos" (Artículo 109). Por su

8 Los resultados de los procesos de evaluación docente serán dados a conocer - por escrito- al docente, por parte del superior inmediato. En caso de inconformidad 0 incongruencia, el docente "podrá presentar recurso de reposición dentro de los cinco (5) días siguientes a la comunicación del resultado de la evaluación. La respuesta que se dé a dicho recurso constituye el pronunciamiento definitivo sobre la evaluación" (p. 29). En todo caso, los resultados y decisiones finales de la evaluación serán registrados en la hoja de vida del docente. Finalmente, como consecuencia la evaluación y en lo relativo al reconocimiento de la labor docente, la Universidad "podrá conceder al docente que haya prestado notables senvicios a la Universidad durante diez (10) años como mínimo y que se haya distinguido por su colaboración, lealtad y eficiencia alguna de las siguientes condecoraciones: Escudo de la Universidad (Primera categoría: en diamantes y oro; Segunda categoría: en oro; Tercera categoría: en plata); Placa de HONOR AL MÉRITO"; por otra parte, allende los reconocimientos y distinciones mencionados, la Institución concede, a juicio del Consejo Superior, estímulos e incentivos a los docentes que se hayan "distinguido por sus aportes académicos a la Universidad y a la comunidad en general"; tales estímulos pueden estar representados en "periodos sabáticos de estudio, pasantías remuneradas en universidades nacionales 0 extranjeras y cualquier estímulo de carácter económico 0 de otra índole" (p. 33). parte, el documento señala la relevancia de unas normas que garanticen la convivencia y el desarrollo armónico de las actividades:

[...] el reglamento es como una carta de navegación para guiar la vida cotidiana de la comunidad estudiantil, orientar los asuntos concernientes a la responsabilidad y al modo de actuar y procede de la vida universitaria; a encontrar la forma de resolver adecuadamente las dificultades y solucionar satisfactoriamente en los problemas que se puedan presentar (2005, p. 22).

Derechos y obligaciones estudiantiles están atravesados por prácticas evaluativas: conocer las normas de comportamiento que rigen a la comunidad; conocer la oferta curricular del programa; conocer las normas y criterios por los que será evaluado su aprendizaje; ser evaluado justamente e informado en los plazos previstos; acceder a reconocimientos, estímulos y ayudas; participar en actividades académicas establecidas, hacer buen uso de medios y mediaciones que cualifiquen su proceso académico, informar oportunamente a sus padres, responsables o acudientes, sobre los avances y resultados de su desempeño académico (pp. 34-36).

Las políticas de ingreso a la Universidad constituyen en cuanto tales, procedimientos evaluativos, dado que prescriben los requisitos sine qua non es posible acceder siquiera al proceso de admisión: selección, "proceso mediante el cual la Universidad evalúa las condiciones humanas y académicas del aspirante, para ingresar, según el perfil y exigencias del programa al cual aspira"; admisión, proceso de aceptación del candidato, tras haber cumplido con los requisitos demandados por la institución o el programa. La norma señala otros procedimientos de ingreso o retorno a la Universidad: ad- 
misión por transferencia externa e interna y reintegros; procesos todos cuyas decisiones conducentes a admisión o exclusión, pasan por modalidades evaluativas (pp. 39-46).

El Reglamento se ocupa (Título VII) de las modalidades de evaluación, exámenes y calificaciones. Por evaluación del aprendizaje entiende: "el modo pedagógico de evidenciar el desarrollo formativo alcanzado por el estudiante de acuerdo con las capacidades y competencias propuestas en la malla curricular del programa; permite al estudiante aprender conscientemente y mejorar en su proceso formativo" (p. 73). El proceso evaluativo se orienta en la Universidad, por "el principio de desarrollo humano integral", que se compone de diversos procedimientos: autoevaluación -la que produce el estudiante sobre su propia formación y sobre su gestión educativa-; coevaluación -la que se realiza entre varios estudiantes, para enriquecer y compartir las experiencias académicas y formativas y verificar sus avances, logros y resultados-; y heteroevaluación efectuada por el sobre el aprendizaje de los estudiantes-. Algunas prácticas evaluativas específicas prescritas por el documento son:

- Examen de suficiencia. Evaluación que una unidad académica efectúa -previa autorización del respectivo Consejo- a un estudiante, con el propósito de "evidenciar si es competente en los conocimientos, aplicaciones, estrategias y fines exigidos en una determinada asignatura, aprobarla y continuar el desarrollo curricular. La nota aprobatoria la determinará el reglamento particular de cada Facultad". Se autoriza, según reglamentación máximo el 50\% de las asignaturas del programa (p. 76).

- Evaluaciones progresivas (pp. 76-77): parciales, cuyo propósito es:
[...] determinar el avance en el proceso de aprendizaje y el esfuerzo formativo del estudiante, en su trabajo independiente y en el acompañado por el docente. Pueden consistir en trabajos, ensayos, lecturas, ejercicios, participaciones en clase, investigaciones personales y en grupo, pruebas orales o escritas, que el profesor juzgue conveniente realizar durante el período lectivo. El resultado sumativo y acumulativo de los mismos da lugar a la "nota o calificación de curso", cuyo valor es del setenta por ciento $(70 \%)$ de la nota final.

\section{- Exámenes finales:}

[...] evaluaciones que se realizan en la culminación del curso, sobre la totalidad de la asignatura como forma de evaluar el logro de los propósitos de aprendizaje, dentro de las fechas establecidas en el Calendario Académico. Su valor ordinariamente es del treinta por ciento $(30 \%)$, salvo cuando se integra con evaluaciones diarias, que sumadas respondan al ciento por ciento $(100 \%)$, como en las teórico-prácticas.

- Exámenes supletorios, "son los presentados por un estudiante cuando por enfermedad certificada por el Servicio Médico de la Universidad, caso fortuito o calamidad doméstica, debidamente comprobados, no se han presentado los exámenes finales o parciales en la fecha determinada.

- Exámenes de validación. "Son las pruebas presentadas para homologar asignaturas cursadas en otra institución de educación superior o en otro programa [...]. Para su aprobación se requiere una nota mínima de tres cinco (3.5)" (p. 78).

- Exámenes preparatorios.

Son las pruebas presentadas por los estudiantes, en forma oral, escrita u otra 
modalidad, según el reglamento de cada programa, sobre los campos de formación o áreas académicas establecidas por las Facultades. Por lo general, se presentan al finalizar los estudios, en las fechas que determine cada Facultad en su reglamento. La forma de evaluación y las fechas fijadas por la respectiva Facultad se deben informar a los interesados. Para su aprobación se requiere una nota mínima de tres cinco (3.5) (pp. 78-79).

- Exámenes de habilitación.

Son las pruebas presentadas para recuperar una asignatura cursada y perdida, mediante un examen, curso de habilitación, o recuperación de logros, en las fechas fijadas en el Calendario Académico. La evaluación comprende toda la asignatura. Si el estudiante reprueba la habilitación debe cursar nuevamente la asignatura (p. 79).

- Recuperaciones. Actividades correspondientes al proceso de complementación del trabajo desarrollado por el estudiante, de acuerdo con los criterios establecidos para la evaluación integral, desde la perspectiva de obtener todos los logros demandados en su campo de formación profesional.

- Asistencia a clases. La asistencia es de obligatorio cumplimiento y responsabilidad del estudiante; ésta se controlará durante la clase. . "El estudiante que deje de asistir al veinte por ciento $(20 \%)$ de las clases dictadas en una asignatura, la perderá por fallas y será calificada con cero cero (0.0)" (pp. 83-84).

- Calificación.

9 El tiempo controlable por el docente es el tiempo presencial. "En caso de enfermedad, certificada por el médico de la Universidad, calamidad doméstica o fuerza mayor debidamente comprobada, el estudiante puede solicitar al Consejo de Facultad la anulación 0 dispensa de las fallas" (p. 84).
[Es] la expresión cuantitativa y cualitativa de las evaluaciones. Se expresan en unidades y décimas, desde cero cero (0.0) a cinco cero (5.0). La calificación mínima aprobatoria para cualquier asignatura es de tres cero (3.0), excepto las de validación, homologación y exámenes preparatorios que debe ser de tres cinco (3.5) (p. 80).

La cuantificación de la evaluación integra todos los procedimientos evaluativos realizados en cada periodo académico ${ }^{10}$ : pruebas rápidas (quizzes), evaluaciones parciales, trabajos, interrogatorios en clase, evaluación del trabajo independiente, notas de investigación, ejercicios, todo trabajo presencial o independiente del estudiante, de modo que las pruebas parciales de todo tipo, sumadas, constituyan el $70 \%$ de la nota final:

[...] el examen final vale el treinta por ciento (30\%), en las asignaturas teóricoprácticas. En las asignaturas prácticas, laboratorios, clínicas y similares la calificación final es la sumatoria de todas las evaluaciones parciales realizadas durante el período académico y equivale al ciento por ciento (100\%) (p. 81).

El sello determinante de los logros, estancamientos o retrocesos, de las inclusiones o exclusiones, de las aprobaciones o reprobaciones los constituyen los certificados de calificaciones, de constancias de matrícula, de paz y salvo, de conducta, de permanencia en la Universidad, de asistencia, de Grado, que expide la institución por solicitud de los

10 Por derecho propio, el estudiante debe conocer sus calificaciones antes de ser registradas definitivamente; ello con el objeto de "impugnarlas y pedir su revisión y corrección, si fuere el caso". Este procedimiento "debe hacerse por escrito en el término de los cinco (5) días hábiles siguientes a su publicación [...], por la Secretaría de la Facultad" (p. 82). El estudiante es, en todo caso, responsable de solicitar oportunamente sus calificaciones y de llevar su respectivo registro. 
interesados; ellos sintetizan las prácticas evaluativas y cifran, a perpetuidad, a los sujetos, constantemente atravesados y confinados por la maquinaria examinatoria (Foucault, 1984). Finalmente, se puede señalar que la graduación -su ritual, pomposidad y ceremoniosidad-es el sucum de los procedimientos evaluativos, es el examen del examen o la evaluación de la evaluación -meta-evaluación se dirá en la actualidad-. Es la ratificación última, de que un estudiante ha logrado sortear con éxito todas las acciones, etapas y procesos de su formación, por lo cual se le reconoce habilitado y capacitado para el ejercicio idóneo de una profesión. Las diversas modalidades de grado, en la USTA suponen naturalmente condiciones y posibilidades diversas de evaluación.

El título es el reconocimiento expreso de carácter académico, que la Universidad otorga al estudiante que culmine y apruebe satisfactoriamente un programa académico ofrecido por la institución por haber logrado los objetivos de formación previstos para el programa y adquiridas las competencias para el ejercicio de la disciplina o profesión, y cumplidos los requisitos exigidos por ella y por la Ley. Tal reconocimiento se hará constar en un diploma y en el acta de grado (pp. 97-98).

Al título se opta toda vez que se haya cumplido con todos los requisitos académicos; cursado y aprobado todo el plan de estudios; aprobado el trabajo o el examen de grado; estar a paz y salvo; y, certificar una documentación determinada (pp. 98-99). El acto público de reconocimiento del cumplimiento de los requisitos y la entrega del título se concreta mediante la ceremonia de graduación; reconocimiento y aceptación social de los recién formados.

\section{Evaluación docente como estrategia para el mejoramiento continuo}

La evaluación docente es considerada como estrategia para el mejoramiento continuo de la calidad académica; por ello se debe desarrollar en el marco de las políticas institucionales y del PEI. La práctica evaluativa:

[...] debe ser una herramienta que apoye los procesos de mejoramiento docente y contribuya eficazmente con el logro de los objetivos de la calidad de la educación y de los servicios que ofrece la institución [...] Debe tener un momento y un contexto determinados, y como tal, debe darse bajo esas condiciones (USTA, 2006, p. 5).

Como todo proceso, la evaluación docente debe seguir unos pasos relacionados entre sí, no necesariamente secuenciales o evolutivos: preparación o alistamiento; desarrollo e implementación; análisis de resultados y acuerdos de mejoramiento; seguimiento de resultados. La puesta en marcha de la maquinaria evaluativa supone la disposición de medidas conducentes al mejoramiento de la calidad de los docentes ${ }^{11}$.

11 Referente a los usos de la evaluación docente, el documento señala: "Fundamentalmente debe usarse como herramienta para acercar al docente a la universidad y para definir acciones de mejoramiento en las debilidades detectadas. Medidas que deben convertirse en acuerdos sujetos a verificación de logros, avances y resultados; Para hacer estudios sobre los avances en calidad a partir del mejoramiento de los docentes; Para tomar decisiones respecto de los tipos de vinculación y contratación; Para diseñar programas de mejoramiento docente promovidos por la universidad y para beneficiarse de los mismos, en la condición de docente. Para los procesos de promoción y estímulo previstos en el escalafón docente de la universidad; Para hacer estudios comparativos entre facultades y entre programas de la Universidad y con otras instituciones de educación SUPERIOR. Para la toma de decisiones en relación con el mejoramiento docente. Para tomar decisiones respecto de la continuidad del docente en la universidad, luego de agotar la etapa de acuerdos de mejoramiento y la verificación de los mismos" (p. 8). 
El instrumental por el cual se efectúa el proceso de evaluación docente consta de:

- Unos formatos de evaluación para sistematización de la información: formulario de evaluación de estudiantes, de docentes por parte del director de la unidad académica y de autoevaluación docente.

- Unos cuadros de reporte: estadística de docentes; estadística de las asignaturas; consolidado por puestos; promedio general; reporte de evaluación del docente por el decano; reporte de autoevaluación docente; reporte de promedios ponderados por facultad; consolidado institucional por calificación. Este proceso evaluativo es permanente y periódico, se replica cada semestre para todos los docentes de la institución.

La política de evaluación docente prescribe el procedimiento de vigilancia de la evaluación, el monitoreo de la evaluación, la evaluación de la evaluación, o mejor, los procesos de meta-evaluación: lega a la Unidad de Planeación y Perfeccionamiento Docente, adscrita a la Vicerrectoría Académica, la coordinación del proceso, el seguimiento y verificación de su complimiento. “Una vez culminada la captura de la información y la entrega de reportes de resultados, la Unidad analizará el proceso y sus avances y dificultades, apoyado en un comité que dará sus recomendaciones al respecto". Finalmente, el documento señala que es responsabilidad de la Vicerrectoría Académica General la integración de un Comité de Monitoría a la Evaluación de Docentes y la determinación de sus funciones, siendo una de ellas "recomendar los ajustes del caso a los instrumentos y al proceso y alcances de la evaluación".

\section{Acreditación y Registro Calificado como prácticas evaluativas}

Desde la promulgación de la Ley 30 de 1992, cuyo espíritu y mandato versan sobre la garantía de la calidad con que se imparten los estudios en la educación superior en Colombia, para preservar derechos legítimos que en esta materia tienen los usuarios del sistema de educación superior y la sociedad en general (CNA, 1997), las instituciones entraron en la moda de la práctica evaluativa en pos de alcanzar ese propósito, a nivel institucional y de sus programas académicos.

Los procedimientos que acompañan la acreditación se han constituido en entes agenciadores-evaluadores (entidades de control) que meta-evalúan las prácticas y desde sus recomendaciones y orientaciones promueven la obtención o no del reconocimiento de alta calidad, para los programas con extensión a la generalidad de su vida académica. Al describir el curso de los procesos de acreditación, se deben distinguir dos escenarios: la institución y los programas.

Los procedimientos de acreditación buscan mejorar la calidad de manera permanente en los procesos de docencia, investigación y proyección social. Además, es un proceso mediante el cual el Estado da fe pública y garantiza a la sociedad que las instituciones de Educación Superior y sus programas académicos cumplen con los más altos requisitos de calidad.

La calidad en la Educación Superior está orientada a promover la competitividad, la pertenencia, eficiencia y eficacia de los programas, frente a los retos de un mundo 
globalizado por el conocimiento. La acreditación es un proceso de carácter voluntario, al cual se someten los programas y las instituciones para garantizar sus niveles de calidad.

La USTA ha lanzado su apuesta por la acreditación tanto de programas como institucional; algunas facultades han ingresado en dichos procedimientos, han realizado las gestiones pertinentes y en consecuencia, sus programas han obtenido reconocimiento (Acreditación de Alta Calidad), por parte del MEN: Filosofía, Derecho, Contaduría Pública, Economía, Ingeniería Electrónica, Ingeniería Mecánica, Psicología.

En lo relativo a la acreditación institucional, "el acto por el cual el Estado adopta y hace público el reconocimiento dado por los pares académicos a la comprobación que efectúa una institución, sobre la calidad de sus programas académicos, su organización y funcionamiento y el cumplimiento de su función social" (CNA, s.f., p. 1), la Universidad ha satisfecho las exigencias legales, ha sorteado diversas etapas (verificación de condiciones iniciales, autoevaluación y evaluación externa) en procura del concepto definitivo y definitorio del proceso.

Por otra parte, para poder ofrecer y desarrollar un programa de formación técnica profesional, tecnológica, y profesional de pregrado o de especialización, nuevo o en funcionamiento, se requiere obtener Registro Calificado de éste, por lo que al Gobierno Nacional le corresponde su reglamentación. Para tal evento, las instituciones de Educación Superior deberán demostrar el cumplimiento de las condiciones mínimas de calidad (Decreto 2566 de 2003). Esta jurisprudencia conceptúa (Artículo 22) el re- gistro como el reconocimiento estatal “del cumplimiento de las condiciones mínimas de calidad para el adecuado funcionamiento de programas académicos de educación superior, mediante su incorporación en el Sistema Nacional de Información de la Educación Superior-SNIES y la asignación del código correspondiente".

\section{Otros documentos que prescriben formas evaluación \\ educativa}

Las políticas institucionales que orientan la vida universitaria proceden de los cuerpos colegiados que las prescriben y que determinan las condiciones reales para su puesta en funcionamiento. Las formas evaluativas, que tras el análisis de un prolijo conjunto de normativas, se presentan seguidamente, describen un proceso -desde su génesis hasta su aprobación y concreción- que atraviesa diferentes umbrales, son sometidos a análisis rigurosos (evaluación) y, tras sortear estas atapas, pasan finalmente a sanción -evaluación: aprobación definitiva o desaprobación- por parte del Consejo Académico, Consejo Superior o Consejo de Fundadores, según competencia.

- Acuerdos emanados de los Organismos Colegiados $^{12}$. Las directivas emanadas de los órganos de dirección académica de la Universidad, Consejo Superior, Consejo Académico General y Consejo Académico Particular, explicitan una amplia variedad de formas y prácticas

12 El análisis se funda en los acuerdos producidos por los organismos colegiados. Especial reconocimiento a los integrantes de la Secretaría General por su ingente trabajo de sistematización de los mismos, piezas documentales facilitadas para el presente análisis. 
evaluativas o examinatorias, que regulan la vida universitaria. Muchas de las modalidades evaluativas prescritas -ya sean directas o derivadas de procedimientos valorativos- se reflejan en los documentos institucionales y se concretan en la totalidad de labores -docencia, investigación y proyección social- que acompañan la dinámica institucional.

La siguiente es una analítica de las formas evaluación, agrupadas según modalidades enunciativas: reforma estatutarias; integración de comisiones y comités (desarrollo, evaluación y planeación de las unidades académicas, revisión, modificación y redacción de documentos institucionales...); reglamentación sobre evaluaciones, calificaciones y porcentajes académicos; creación y aprobación de unidades académicas: facultades, programas académicos y unidades de apoyo académico (facultades y programas académicos-pregrado, programas académicos de posgrado-especializaciones, programas académicos-posgradomaestría, creación y aprobación de otros centros interdisciplinarios y unidades académicas, establecimiento y suscripción de convenios y otros organismos); reorganización, reforma... de unidades académicas; prácticas punitivas y sancionatorias; prácticas de gratificación y reconocimiento; políticas de admisión y vinculación docente; adopción de procedimientos de acreditación, etc.

- La política de admisión como práctica evaluativa. El documento que explicita las políticas de admisión de estudiantes en la Universidad Santo Tomás (s.f.), en consonancia con el Reglamento Estudiantil (Capítulo IV), prescribe en sus regulaciones un conjunto de prácticas evaluativas (inclusión-exclusión, acepta- ción-rechazo, validación-invalidación), con que se busca (p. 2):

- Admitir a sus programas académicos personas que se ajusten al perfil del estudiante tomasino. La persona seleccionada es consciente de que ella se constituye en el principal agente responsable del proceso de formación integral. Se buscan personas con un alto grado de responsabilidad y un profundo sentido ético y espiritual; con un claro compromiso social y potencialidad para llegar a ser futuros agentes de cambio y de transformación personal y social y con un conocimiento básico de lo que la Universidad Santo Tomás representa para el país como primer claustro universitario colombiano.

- La regulación prescribe criterios para admisión e inscripción. El proceso para inscripción que inicia con la adquisición del respectivo formulario, al que se deben anexar unos documentos. Posterior a la inscripción se desarrolla el procedimiento de selección a través de una entrevista personal y en algunos casos, la presentación de exámenes específicos, según facultades ${ }^{13}$.

- Informe Ejecutivo del Proceso de Acreditación Institucional. Los procesos de autoevaluación han acompañado buena parte de la vida institucional, posibilitando la toma de conciencia sobre su propia identidad. El documento, tras abordar un prolijo análisis sobre el con-

13 "El resultado de las pruebas del ICFES tiene un valor del $50 \%$ y el de la entrevista [examen] otro $50 \%$. Una vez se consolida esta información se define la lista de admitidos y se informa a los estudiantes mediante la publicación de los resultados [... ]" (p. 28). El proceso de admisión finiquita con la firma de la matrícula: "contrato por medio del cual se adquiere la calidad de estudiante de la Universidad Santo Tomás" (p. 32). 
texto institucional (naturaleza, historia, proyecto, organización, interacción, gestión, infraestructura y recursos), se ocupa del proceso de autoevaluación institucional (marco de referencia, modelo evaluativo, metodología y etapas); insumos presentados con los demás requisitos y documentos institucionales, con la intencionalidad de solicitar la visita de pares académicos para Acreditación Institucional.

A fin de concretar los procesos de calidad, la Universidad implementó (junio de 2002) el Sistema de Gestión de la Calidad, cuyo objetivo se orienta a la creación de "una cultura organizacional enfocada a gestionar las actividades mediante la gerencia por procesos [...], el Sistema se orienta a fortalecer el uso de datos e información confiable para la toma de decisiones y el mejoramiento continuo de los procesos" (USTA, 2007, p. 69). El Sistema constituye uno de los mecanismos de seriación y ciframiento, en la medida en que incluye procesos de dirección, académicos y de apoyo, cada uno de los cuales se describe a través de fichas de caracterización de procesos, productos y flujogramas, que identifican con claridad las actividades, los responsables, los documentos, los formatos, y los modos de desarrollo de las acciones. “Cada proceso mide su gestión por medio de indicadores orientados a verificar el cumplimiento de los objetivos de calidad que han sido definidos de acuerdo con las directrices de la Política de calidad de la Universidad" (p. 70).

- Hacia un modelo de evaluación para la Universidad Santo Tomás. Este documento (USTA, 2003) describe un modelo de evaluación integral, cuantitativo-cualitativo, basado en un enfoque sistémico e inspirado en la misión; opera sobre la base de unos principios (investigación, flexibilidad, responsabilidad, realismo, mejoramiento, autonomía, ético, creatividad, cooperación y liderazgo) y unos criterios (universalidad, integralidad, equidad, idoneidad, responsabilidad, coherencia, transparencia, pertinencia, eficacia, eficiencia) ${ }^{14}$.

Sobre el modelo de evaluación, el documento indica que éste no puede ser ajeno al contexto educativo, por tanto, "la concepción y práctica evaluativa ha de ser coherente en su concepción con dichos fines y principios de la educación que requiere el país para los fines del desarrollo a todo nivel" (p. 9). Asimismo, prescribe (p. 14) los objetivos orientadores de la autoevaluación institucional:

- Promover la actualización y el mejoramiento académico de los programas de la Universidad, a través de la iniciación de un proceso de autoevaluación para la autorregulación con fines de construcción de calidad y acreditación formal.

- Orientar a los diferentes programas e instancias de la Universidad sobre los diferentes procesos y actividades

14 Los objetivos del modelo son: "Generar una cultura de evaluación integral en la Universidad, orientada sobre la vida académica y administrativa, que permita en forma permanente construir procesos de calidad integral; Promover la integración y participación de la comunidad universitaria a partir de reconocer desde procesos evaluativos, posibilidades de crecimiento y cualificación del sistema educativo de la Institución; Asumir la evaluación como un espacio trascendente que permite cohesionar los fines misionales de la Institución y elevar en forma permanente los estándares de calidad; Garantizar la mejor calidad en las acciones académicas de la Universidad, a partir de reconocer debilidades e intervenir los problemas con planes de regulación y mejoramiento continuo; Fomentar la cultura de los proyectos de autorregulación y mejoramiento continuo en todas las dependencias académicas e instancias administrativas de la Universidad, como una forma de gestionar las intencionalidades propias del Modelo de Evaluación y regular así, la calidad de manera integral" (p. 11). 
propias del proceso de autoevaluación y regulación académica con fines de acreditación y construcción de calidad.

Los objetivos mencionados se orientan a la cobertura de todas las acciones que desarrolla la institución -a través de sus unidades y agentes- en lo relativo a la docencia, investigación y proyección social.

- Evaluación modular. Desde la adopción de la metodología modular en la Facultad de Derecho, la evaluación ha hecho presencia y se ha venido modificando paulatinamente, ampliando su radio de acción y perfeccionando su instrumental, con el objeto de convertirse en dispositivo que acompaña todos los procesos de enseñanza-aprendizaje.

Tres documentos se lograron identificar:

- Módulos 13 (USTA, 1984), el cual describe y conceptualiza la evaluación desde la metodología de la analítica, como "el proceso consistente en determinada hasta qué punto los objetivos educativos específicos se están logrando" (p. 10), tal proceso se orienta a comprobar la congruencia existente entre los resultados y los objetivos.

- Módulos 25 (USTA, 1986), documento que reconceptualiza la evaluación "proceso permanente e integral de la función educativa, que busca conocer, retroalimentar y mejora del desarrollo de la actividad docente"; señala unas modalidades y tipologías evaluativas (diagnóstica, formativa, sumativa; escrita, oral; exámenes preparatorios, evaluaciones de trabajos de grado...); manifiesta ventajas y desventajas de cada modelo y hace recomendaciones para tener en cuenta al momento de evaluar.

- Módulos 54 (USTA, 2006) constituye un replanteamiento definitivo (actualmente) de los procedimientos de valoración del trabajo académico de los estudiantes. El documento se ocupa de desarrollar los procedimientos específicos del sistema modular:

[...] en especial en lo concerniente al examen modular [que según el Reglamento de la Facultad tiene un Valor del $30 \%$ de la nota definitiva], cuya modalidad ha pasado de la prueba objetiva, tipo "test", un examen oral, argumentativo y propositivo sobre casos específicos de la realidad (pp. 7-8).

Así mismo, presenta las pautas generales para el desarrollo de la evaluación señalar los objetivos del curso y no apartarse de ellos; pactar con los estudiantes los criterios de evaluación y permanecer fieles a este acuerdo; y, retroalimentar oportunamente los resultados-; pone en el centro esta práctica evaluativa "el autoaprendizaje del estudiante", proceso en el que "la evaluación no es control", tampoco es "exclusión de lo divergente" $\mathrm{y}$, por tanto, constituye un procedimiento perpetuo de autoevaluación, de manera que, "evaluar es evaluarse".

\section{Complementos directos de la memoria histórica institucional}

La caracterización e historización de las formas evaluación educativa finiquita con una sucinta reconstrucción histórica, a partir muestras testimoniales de algunos docentes y directivos, quienes las han vivido en carne propia, como sujetos pasivos o activos de diversas prácticas y modalidades evaluativas, 
cuando eran estudiantes y en la actualidad como evaluadores (docentes o directivos) y en cuanto tales, han sido testigos directos de la dinámica evaluativa que ha acompañado la actividad universitaria ${ }^{15}$.

El objetivo de recoger estos testimonios fue un intento de reconstrucción de la memoria histórica institucional sobre modalidades de evaluación educativa, dada la adolescencia de documentos y registros institucionales que dieran cuenta de dichas prácticas (p. 5). Los siguientes temas sintetizan el análisis de las entrevistas; el punto de partida: una aproximación al concepto evaluación.

- Es parte del proceso educativo: como momento de inicio o final. Como elemento final de un proceso, luego de que se han dictado un conjunto de contenidos, temas, problemas, unidades... y, al final se recopila todo ese proceso; es lo que antiguamente se conocía como exámenes finales, que se realizaban luego de un proceso en que se había visto, analizado, discutido, asumido, una temática determinada; pero, además, se podía considerar como un momento de inicio

15 Para la reconstrucción de la memoria histórica de las formas evaluación educativa en la USTA, se acudió a entrevistas (semiestructuradas) y conversaciones informales con varias personas: fray Pedro J osé Díaz Camacho O. P. (Vicerrector Académico), profesores Eudoro Rodríguez Albarracín, Alfredo Gil Rico (Departamento de Humanidades), Carlos J ulio Flórez Márquez (Director del Departamento de Humanidades), Teresa Hougton (Facultad de Filosofía), Martín Rodríguez (Facultad de Economía), Alberto Cárdenas (Facultad de Derecho), Gregorio Clavijo (Facultad de Sociología), Luis López (ex-decano) y Héctor Fabio Santamaría (Secretario General).

Estas herramientas se usaron como mecanismos de recopilación de la historia. Es importante aclarar que la dinámica conversacional con el entrevistado permite un diálogo en el que es posible ir construyendo en forma flexible y circular la información, a partir de unas preguntas elementales que generan el diálogo entre los actores. Los temas centrales de las preguntas están relacionados con una reconstrucción histórica a partir de la experiencia personal sobre la evaluación en la universidad. para planeación y para valorar el desenvolvimiento del curso, el desarrollo del proceso, los avances realizados, las dificultades presentadas, etc.

- Es la valoración cuantitativa y cualitativa del proceso educativo. En principio, consistía en un conjunto de preguntas, a las que se asignaba un puntaje con base en el cual se obtenían las conclusiones que daban lugar a las calificaciones. Era más cuantitativa que cualitativa.

- En la evaluación predominó (y aún se evidencia) un enfoque cognoscitivo, había un predominio fijado en los contenidos -contenidos, una serie de temas, unos programas que se desarrollaban-, al final, sobre todo, ese conjunto de cuestiones y con base en los objetivos se obtenía la evaluación; pero no había evaluación, por ejemplo, de actitudes, de valores, de diagnóstico o dinámica de grupos, autoevaluación, coevaluación, etc.

- Los entrevistados distinguen dos momentos: primero, lo padecido como estudiante; y luego, lo ejecutado en el ejercicio profesional. En la época de estudiantes, en algunas cátedras se acostumbraba a realizar dos parciales, desarrollados a partir de ciertos contenidos -con determinados porcentajes- y un examen final. Tanto en parciales como en el examen final se enfatizaban las preguntas abiertas, con algunas excepciones de preguntas cerradas. En otras áreas había profesores que pedían trabajos escritos sobre una temática específica, como una especie de ensayo o, más que ensayo, consistía en el desarrollo de un tema, en el que se sustentara tanto el contenido de la temática -como el punto de vista del estudiante.

- El estilo de evaluación depende, en buena medida, del estilo de docencia y 
de las características del profesor. A lo largo de la vida académica se encuentran profesores para todo: unos acentuaban lo memorístico, otros de tipo reflexivo (hermenéutico). Algunos profesores introdujeron exámenes distintos; por ejemplo, los test, que en principio, dada su novedad, no produjeron buenos resultados.

- Las prácticas evaluativas, se sintetizaban en: primer parcial, segundo parcial y examen final. Si contestaban bien estas pruebas, los estudiantes pasaban sin ningún problema. La evaluación era tradicional, se basaba en objetivos, contenidos, programas y al final las preguntas escritas con algunas variantes (exámenes orales). Esa vieja costumbre ya no se da, ahora la evaluación va acompañada de la asistencia de los estudiantes, de la actitud, de la participación en clase, de consultas, de trabajos, de exposiciones, de ensayos, de parciales, de procesos de autoevaluación u coevaluación y, de otras tantas prácticas evaluativas.

- Anteriormente, no se desarrollaban procesos de autoevaluación, ni coevaluación, simplemente, el profesor evaluaba y era una evaluación vertical, según el modelo que criticaba Freire (educación bancaria); actualmente, muchos docentes utilizan procedimientos de autoevaluación, sin embargo, este ejercicio es muy subjetivo y riesgoso. Una faceta lamentable de la evaluación es su uso (de manera consciente), como un ejercicio de imposición y castigo.

\section{A MOdo de CONCLUSIón}

La trama evaluativa ha hecho presa de saberes, sujetos, instituciones, políticas, proyectos, acciones, etc., de las comunidades académicas y de la sociedad en su conjunto. En la Universidad, las mallas del poder evaluativo se han extendido sobre todos los sujetos e instancias constructores de la verdad y constituidos por ella: los docentes con cualquier modalidad de vinculación; estudiantes de todos los programas presenciales, jornadas y modalidades; administrativos y directivos de unidades académicas, que se constituyen en evaluadores, pero, al mismo tiempo, en orientadores de procesos y como tal, agentes sometidos permanentemente a procedimientos evaluativos.

La analítica realizada sobre documentos institucionales condujo a la identificación de un cúmulo de formas evaluación que de modo directo o indirecto inciden en la educación que ofrece la Universidad y que implican, necesariamente, al conjunto de los individuos, acciones y procesos propios de la comunidad académica: vigilancia del cumplimiento de políticas; regulación $\mathrm{y}$ control del desarrollo de procesos y de la gestión; aprobación-reprobación de saberes, sujetos, acciones, procesos, etc.; reforma de estatutos, reglamentos, políticas, proyectos, etc.; concesión-negación de méritos y reconocimientos; creación, extensión, supresión de sedes, programas, unidades académicas; análisis y estudios de factibilidad; resolución de procesos académicos y disciplinarios; escogencia de opciones; toma de decisiones y determinaciones; ejecución de acciones, nombramientos y remociones de funcionarios; direccionamiento de procesos; gestión, organización, desarrollo de actividades y programas; aplicación de políticas; elección, admisión, promoción, graduación (de estudiantes); aprobación de planes de estudio y reformas de programas académicos; aprobación del calendario académico, de los sistemas de evaluación y los métodos generales 
de docencia e investigación; diseño de mecanismos para la evaluación, promoción y especialización (del cuerpo docente); estímulo y promoción de producción y publicaciones (fruto de docencia e investigación); supervisión de proceso y sistemas de registro académico; promoción y ascenso o exclusión de sujetos (administrativos, docentes, directivos, estudiantes...); asesorías sobre diversas actividades relativas a las funciones sustantivas; dirección de proceso evaluativos (de docentes, de discentes, de gestión...); evaluación, reforma y actualización de currículos, programas, planes de estudio, etc.; auspicio y suscripción de convenios ínter e intra-institucionales; organización de procedimientos de inducción (docentes, estudiantes y personal administrativo nuevo); direccionamiento de procedimientos de matrícula; organización y puesta en marcha de criterios de admisión y selección de agentes educativos; determinación de las políticas y pautas para el ejercicio de las funciones sustantivas (docencia, investigación y proyección social); planeamiento y ejecución de políticas de bienestar y pastoral.

Prácticas evaluativas y correlativas a la acción académica: evaluación de programas e institución con fines de acreditación, evaluación institucional y de programas como proceso de mejoramiento continuo, evaluación curricular, evaluación de las funciones sustantivas, mejoramiento académico de programas con fines de registro calificado, evaluación de gestión, evaluación docente, evaluación sumativa, formativa, diagnóstica, propositiva, de intervención, autoevaluación, heteroevaluación, coevaluación, metaevaluación, evaluación externa, etc.; y formas específicas de evaluación de los procesos de enseñanzaaprendizaje: parciales (orales y escritos), reseñas de documentos, exposiciones, ponencias, debates, elaboración de ensayos, exámenes finales, experimentos, pruebas, cuestionarios, controles de lectura, revisión bibliográfica y de documentos, composiciones, consultas, preparación de actividades, seminarios, discusiones, lecturas, talleres, estudios de caso, análisis de prensa, análisis de problemas, previas, trabajos individuales $\mathrm{y}$ grupales, protocolos, proyectos, informes salidas de campo, grupos de discusión, seguimiento del alumno, ejercicios en clase, actividades investigativas, mapas conceptuales, crucigramas, sopas de letras, elaboración de cartillas y folletos, monitorías, guías de trabajo, pruebas iniciales, intermedias y finales, creatividad, disposición, entusiasmo e interés del estudiante, construcción colectiva, asistencia (clases y asesorías), preguntas y respuestas, elaboración de fichas y ficheros, quizes, informes de avance de trabajos y proyectos, informes clínicos, intervención y participación en clase, adquisición de conceptos y destrezas, elaboración de memorias, etc.

Las modalidades evaluativas descritas son manifestación de la presencia y de la relevancia de los procesos evaluativos que han conquistado y colonizado la cotidianidad de los sujetos y las instituciones; y, que como tal se han constituido en estrategias que todo lo gestionan: producen saberes, determinan relaciones de poder y fabrican subjetividades. Dado que, en últimas, esta indagación se ocupó de una descripción (escueta) de las formas evaluativas -en la historia reciente de la Universidad Santo Tomás-, se dejan diversos espacios abiertos para indagaciones futuras, toda vez que será de capital relevancia señalar cómo se ha configurado en un saber; cómo ha operado en tanto instancia de poder; y qué tipos de sujetos modela o fabrica toda vez que son presa sus redes. 


\section{REFERENCIAS}

Castro, E. (2004). El vocabulario de Michel Foucault. Buenos Aires: Prometeo.

Congreso de Colombia. Ley 30 de 1992 (28 de diciembre de 1992). Por la cual se organiza el servicio público de la Educación Superior. Bogotá: Diario Oficial No. 40.700. (29 de diciembre de 1992).

Congreso de Colombia. Ley 115 de 1994 (febrero 8). Por la cual se expide la ley general de educación. Diario Oficial Número 41.214. (8 de febrero de 1994).

CNA (1997). Guía para la Evaluación Externa con fines de Acreditación de Programas Académicos de Pregrado. Bogotá: CNA

CNA (Sin fecha). Respuesta a preguntas frecuentes. Recuperado el 8 de julio de 2008 de: http://www.cna.gov.co/cont/ cna/index.htm

Foucault, M. (1970). La arqueología del saber (4a ed.). México: Siglo XXI Editores.

Foucault, M. (1973). El orden del discurso. Barcelona: Tusquets Editores.

Foucault, M. (1980). La verdad y las formas jurídicas. Barcelona: Gedisa Editorial.

Foucault, M. (1984). Vigilar y castigar (9a ed.). México: Siglo XXI Editores.

Grupo de Investigación Docimófilos. (2008, marzo-junio). Complementos directos de la memoria histórica institucional. [Archivo de Audio-Entrevista Docentes]. Bogotá: USTA.

Guyot, V., Marincevic, J. \& Becerra, M. (1996). Los usos de Foucault. Buenos Aires: El Francotirador Ediciones.

Martirena, O. (1995). Michel Foucault: historiador de la subjetividad. México: ITEMSEl Equilibrista.

Morey, M. (1983). Lectura de Foucault. Madrid: Taurus.
Presidencia de la República-MEN. Decreto 2566 de 2003 (Octubre 9). Por el cual se establecen las condiciones mínimas de calidad y demás requisitos para el ofrecimiento y desarrollo de programas académicos de educación superior y se dictan otras disposiciones. Recuperado el 5 de abril de 2008 de: http://www.mineducacion.gov. co/1621/propertyvalue-31213.html

Universidad Santo Tomás (1972). Estatuto Orgánico y Reglamento General. Bogotá: Universidad Santo Tomás.

Universidad Santo Tomás (1999). Proyecto Educativo Institucional. Documento de trabajo. Bogotá: Universidad Santo Tomás.

Universidad Santo Tomás (2001). Proyecto de Investigación Institucional. Bogotá: Universidad Santo Tomás.

Universidad Santo Tomás (2002). Estatuto Orgánico de la Universidad Santo Tomás. Bogotá: Universidad Santo Tomás.

Universidad Santo Tomás (2003). Hacia un modelo de evaluación para la Universidad Santo Tomás. Bogotá: Universidad Santo Tomás.

Universidad Santo Tomás (2004a). Política Curricular. Bogotá: Universidad Santo Tomás. Recuperado de: http://www. usta.edu.co/inf_general/doc_pdf/politica_curricular.pdf

Universidad Santo Tomás (2004b). Estatuto Docente. Bogotá: Universidad Santo Tomás. Recuperado de: http://www.usta. edu.co/inf_general/doc_pdf/estatuto_ docente.pdf

Universidad Santo Tomás (2004c). Proyecto Educativo Institucional-PEI. Bogotá: Universidad Santo Tomás. Recuperado de: http://www.usta.edu.co/inf_general/ pei.html 
Universidad Santo Tomás (2005). Reglamento Estudiantil Pregrado. Bogotá: Universidad Santo Tomás.

Universidad Santo Tomás (2007). Informe Ejecutivo de Autoevaluación con fines de Acreditación Institucional. Bogotá: Universidad Santo Tomás.

Universidad Santo Tomás (Sin fecha). Política de Ingreso. Bogotá: Universidad Santo Tomás. Recuperado de: http://www. usta.edu.co/inf_general/polingreso. html

Universidad Santo Tomás (Facultad de Derecho) (1984). Módulos No. 13. Bogotá: Universidad Santo Tomás.

Universidad Santo Tomás (Facultad de Derecho) (1986). Módulos No. 25. Bogotá: Universidad Santo Tomás.
Universidad Santo Tomás (Facultad de Derecho) (2006). Módulos No. 54. Bogotá: Universidad Santo Tomás.

Universidad Santo Tomás (Secretaría General) (2008). Archivo Documental. Compendio de Acuerdos del Consejo Superior y del Consejo Académico General [Documento Inédito]. Bogotá: Universidad Santo Tomás.

Universidad Santo Tomás (Unidad de Investigación y Posgrados) (2007). Hallazgos.

Universidad Santo Tomás (Unidad de Planeación Académica y Perfeccionamiento Docente) (2006). La evaluación de Docentes como estrategia para el mejoramiento continuo de la Calidad Académica. Bogotá: Universidad Santo Tomás.

Zuluaga, O. (1987). Pedagogía e historia. La historicidad de la pedagogía. Bogotá: Foro Nacional por Colombia. 